\title{
Cesarean Section or Instrumental Delivery: The Best Choice for Mode of Delivery during Second Stage of Labor
}

\author{
Suniti Rawal', Neeta Katuwal2, Sajana Shrestha ${ }^{3}$ \\ Department of Obstetrics and Gynecology, Maharajgunj Medical Campus, Tribhuvan University Teaching Hospital, \\ Institute of Medicine, Kathmandu, Nepal
}

\section{Corresponding author:}

Suniti Rawal, MBBS, MD

Department of Obstetrics and Gynecology, Maharajgunj Medical Campus, Tribhuvan University Teaching Hospital, Institute of Medicine, Kathmandu, Nepal

Email:sunudinurawal@yahoo.com

Submitted : Jun 17, 2020

Accepted : Jul 22, 2020

\begin{abstract}
Introduction

Managing second stage of labour is very crucial. Emergency Cesarean Section (CS) or Instrumental Delivery (ID) is the only choices although complications might occur in both modes of deliveries. The rate of CS is at rise and the fear of failed ID followed by emergency CS with added complications makes the choice more difficult amongst the obstetricians.
\end{abstract}

\section{Methods}

A retrospective study done to assess the maternal and fetal outcome in second stage of labour following ID and emergency CS from April 2018 to March 2019 among the pregnant women having completed 34 weeks of gestations and beyond.

\section{Results}

Of 4761 deliveries, 2537 (53.2\%) were vaginal and 2224 (46.7\%) CS, and amongst all, 133 (2.7\%) were second stage interventions. Out of 133, 78 had ID, 76 were successful and 2 failed. Fifty seven (2.6\%) were second stage CS. Nulliparas $(65.8 \%)$ needed more ID, teenage pregnancy $(6.5 \%)(p=0.04)$ and heart disease $(18.4 \%)(p=0.002)$ were high risk for ID. Complications were more in CS, uterine extensions, $(29.8 \%)$ $(p<0.001)$, urinary complications, $(43.9 \%)(p<0.001)$ and blood loss $(p<0.001)$. Neonatal admission too was higher in CS $(n=29)$ and more so due to respiratory distress $(16 / 29)$.

\section{Conclusion}

Despite of few failed attempted ID, it still proved to be better choice for delivery during second stage, as both maternal and neonatal complications were higher following the second stage CS. Hence, the correct and timely decision for ID addressing the associated risk factors could lead to favorable outcome.

Keywords: Cesarean Section, instrumental delivery, second stage of labour 


\section{INTRODUCTION}

nstrumental deliveries have been adopted for age for delivering of babies during second stage of labour. But now the art of instrumental delivery (ID) is gradually fading ${ }^{1}$ and the trend of cesarean section (CS) is at rise with increasing sophisticated life-style and health awareness. ${ }^{2-5}$

The second stage is the time between full cervical dilatation and birth of the baby, during which the woman has an urge to bear down, as a result of expulsive uterine contractions. ${ }^{6}$ It is said to be prolonged if certain time lapses despite of cervix being fully dilated, pertinent to parity and epidural anesthesia. ${ }^{7}$

Both instrumental as well as emergency CS can be performed in second stage of labour provided the indication and prerequisites are fulfilled for the former.

Once there is arrest of descent or the dilatation in second stage, obstetrician now has to decide either to go for ID or emergency CS. The decision depends on many factors like the Cephalopelvic Disproportion (CPD), Deep Transverse Arrest (DTA), and sometimes fetal distress, poor maternal effort and prophylactic application of instruments but the clinical judgment here has to be prudent. ${ }^{8-10}$

Failure of judgment may leads to grave maternal and neonatal morbidity or mortality. Numerous studies have been documented where emergency CS was performed as first line intervention for second stage rather than ID. ${ }^{11-15}$ However the best method amongst the two is still debatable.

Hence this study was carried out to assess the maternal and fetal outcomes in both types of interventions in second stage of labour.

\section{METHODS}

It was a retrospective study conducted in the Department of Obstetrics and Gynecology, Institute of Medicine; Tribhuvan University Teaching Hospital for from April 2018 to March 2019. All the pregnant women after $34+$ weeks of gestation in second stage of labour having ID or CS were included in the study, whereas patients with intra uterine fetal death (IUFD)/congenitally malformed fetus, placenta previa, multiple pregnancies, posted for elective CS or with previous CS/laparotomy/myomectomy were excluded from the study. Permission from the Department and the ethical clearance was taken from the Institutional Review Committee (IRC) of Institute of Medicine.

The main objective of the study was to assess the maternal and fetal outcome in second stage of labour following ID and emergency CS. From the maternal side, age, parity, gestational age, subfertility and the risk factors for second stage of labour, labour characteristics, indications for ID or CS in second stage of labour and maternal complications were extracted from the records. On the neonatal side, birth weights, Neonatal Unit (NNU)/ Neonatal Intensive Care Unit (NICU) admission, Apgar score were recorded from the files of NNU/NICU.

All the relevant data were collected in the proforma and entered in IBM SPSS version 24. Comparative analysis of categorical and continuous variables was performed using Chi-square and Fisher's exact test and the means of quantitative variables were compared by using student's t-test. The p-value < 0.05 was considered statistically significant.

\section{RESULTS}

A total of 4761 deliveries took place from April 2018 to March 2019, of which 2537 (53.2\%) were vaginal and 2224 (46.7\%) were cesarean section (CS). Of the total deliveries, 133 (2.7\%) patients had second stage intervention, with instrumental deliveries (ID) attempted in 78, of which 76 were successful and 2 failed needing CS.

Of 76 instrumental deliveries, 74(97.3\%) were vacuum and $2(2.6 \%)$ were forceps deliveries. Of the total $2224(46.7 \%)$ cesarean section 57 (2.6\%) were second stage CS

The age of the patient were comparable with the mean of 25 years in both the groups but the gestational age varied and was statistically significant ( $p=0.02)$. Both the ID and CS were found to be more common among nulliparous. Among the women who had ID, 50 (65\%) were nulliparous and $26(34.2 \%)$ were multiparous. Out of those who had CS, 47 (82.5\%) were nulliparous. The result was statistically significant $(p=0.04)$.

Table 1. Maternal demographic profile

\begin{tabular}{lccc}
\hline \multicolumn{1}{c}{ Variable } & $\begin{array}{c}\text { CS } \\
(\mathbf{n}=57)\end{array}$ & $\begin{array}{c}\text { ID } \\
(\mathbf{n}=76)\end{array}$ & $\begin{array}{c}\mathrm{p} \\
\text { value }\end{array}$ \\
\hline Age (year) & 25.02 & 25.19 & 0.27 \\
& $(20-32)$ & $(18-39)$ & \\
Gestational age & 38.77 & 39.37 & 0.02 \\
(week) & $(34-41)$ & $(34-43)$ & \\
Parity & & & \\
Nulliparous & $47(82.5)$ & $50(65.8)$ & 0.04 \\
Multiparous & $10(17.5)$ & $26(34.2)$ & \\
Risk factor & & & \\
Teenage pregnancy & 0 & $5(6.5)$ & 0.04 \\
Advanced maternal age & 0 & $3(3.9)$ & 0.12 \\
Heart disease(RHD+CHD) & $1(1.8)$ & $14(18.4)$ & 0.002 \\
Pregnancy induced HTN & $4(7)$ & $1(1.3)$ & 0.08 \\
Chronic HTN & 0 & $1(1.3)$ & 0.38 \\
Gestational DM & $2(3.5)$ & 0 & 0.10 \\
Thyroid disorder & $3(5.3)$ & $1(1.3)$ & 0.18 \\
Subfertility & 0 & $1(1.3)$ & 0.38 \\
\hline
\end{tabular}


Table 2. Comparison of labour characteristics

\begin{tabular}{lccc}
\hline \multicolumn{1}{c}{ Variable } & CS (n=57) & ID (n=76) & p value \\
\hline PROM & $13(22.8)$ & $8(10.5)$ & 0.09 \\
Onset of labour & & & \\
Spontaneous & $39(68.4)$ & $58(76.3)$ & \\
Induced & $18(31.6)$ & $18(23.7)$ & 0.33 \\
Fetal head position & & & \\
Occipitoanterior & $28(49.1)$ & $76(100)$ & \\
Others & $29(50.9)$ & 0 & 0.000 \\
& & & \\
\hline
\end{tabular}

Twenty six patients were detected to have some or the other risk factors in them, which could have been a contributing factor for ID. Maximum of them (14) $18.4 \%$ were with heart disease, Rheumatic heart diseases (RHD) and Congenital Heart Disease(CHD) combined. This was followed by teenage pregnancy in five $(6.5 \%)$ contrary to three $(3.9 \%)$ who were of advanced age. Ten cases were found to have high risk for CS, of which (4) 7\% were pregnancy induced hypertension (PIH), two (3.5\%) Gestational diabetes mellitus (GDM) and three (5.3\%) of thyroid disorder. There was only one case of heart disease that had second stage CS. Heart disease ( $p=0.002)$ and teenage pregnancy $(p=0.04)$ were found to be statistically significant (Table.1)

Spontaneous onset of labour was present in 39 $(68.4 \%)$ cases which later had second stage CS and $58(76.3 \%)$ had ID. (Table 2).

Maximum number $(29,38.2 \%)$ of ID was done for fetal heart abnormality (tachycardia and/or bradycardia) that were followed by meconium stained liquor $(25,32.9 \%)$. Prophylactic ID in heart disease was done in 10 cases (13.2\%).

In 57 patients, the most frequent indication for second stage CS was non descent of fetal head (44, $77.2 \%)$.$) . Two (3.5\%) had trial of vacuum delivery$ that failed leadina to second stage CS. (Fia 1)

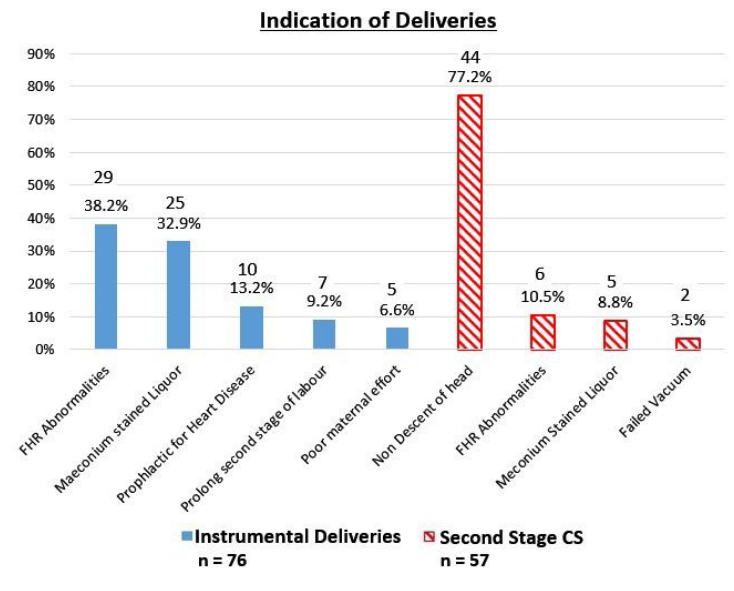

Fig 1. Indications of deliveries
Table 3. Maternal complications

\begin{tabular}{lccc}
\hline \multicolumn{1}{c}{ Variable } & CS $(n=57)$ & ID $(n=76)$ & $p$ value \\
\hline Uterine extensions in & & & \\
CS/ $3^{\text {rd }} 4^{\text {th }}$ degree & & & \\
perineal tear in ID & $17(29.8)$ & $5(6.6)$ & $<0.001$ \\
Urinary complications & $25(43.9)$ & $2(2.6)$ & $<0.001$ \\
Wound complication & $8(14)$ & 0 & $<0.001$ \\
PPH & $2(2.6)$ & $2(3.5)$ & 0.76 \\
Estimated blood loss & & & \\
(ml) $\pm S D$ & $243.86 \pm 80$ & $117.73 \pm 69<0.001$ \\
\hline
\end{tabular}

Maternal complications like uterine extension during second stage CS was noted in 17 (29.8\%) patients. Likewise $3^{\text {rd }}$ and $4^{\text {th }}$ degree perineal tear during ID was seen in $5(6.6 \%)$ cases which was statistically significant $(p<0.001)$.

Urinary complication like prolonged catherization or retention of urine was statistically significant as it was observed in almost half the patients who had second stage CS $(25,43.9 \%)$. Wound complication occurred in $8(14 \%)$ cases of CS which was statistically significant $(p<0.001)$.

Post-partum hemorrhage was identical in both the groups, $2(2.6 \%)$ in CS and 2 (3.5\%) following instrumental delivery. The estimated blood loss was $243.86 \pm 80 \mathrm{ml}$ during CS and $117.73 \pm 69$ in ID which was statistically significant $(p<0.001)$. (Table 3)

The neonatal characteristics and outcome were also assessed (Table 4). The birth weight of the neonate was almost consistent in both the groups which were $3082.98 \pm 397 \mathrm{gms}$ in CS and 3031.76 $\pm 399 \mathrm{gms}$ in ID group ( $p=0.47)$. The APGAR score $<7$ in 5 min was 6 $(10.5 \%)$ in CS and $2(2.6 \%)$ in ID group that was not statistically significant $(p=0.06)$. Admission to NNU and NICU were quite remarkable. About 50.9\%, (29) neonates got admitted following second stage CS and 22.9\% (17) following instrumental delivery, that was statistically significant $(p<0.001)$.

Major reason for NNU/NICU admission in CS group (16/29) was respiratory distress like grunting and tachypnea. Similarly 13/17 neonates following ID also had respiratory distress. One neonate had subgaleal haematoma following failed ID that was followed by second stage CS.

Table 4. Neonatal characteristics and outcome

\begin{tabular}{lccc}
\hline Variable & CS $(n=57)$ & ID $(n=76)$ & $p$ value \\
\hline $\begin{array}{l}\text { Birth weight } \\
\text { (gm) }\end{array}$ & $3082.98 \pm 397$ & $3031.76 \pm 399$ & 0.47 \\
NNU/NICU & & & \\
$\begin{array}{l}\text { admission } \\
\text { APGAR score }\end{array}$ & $29(50.9)$ & $17(22.9)$ & $0.001^{*}$ \\
$<7$ in 5 min & $6(10.5)$ & $2(2.6)$ & 0.06 \\
\hline
\end{tabular}




\section{DISCUSSION}

The second stage of labor is a very important period during delivery where an unexpected problem may occur suddenly to the fetus and parturient. As observed in the current study, 133 of the total 4761 deliveries had second stage complications of which, 57 had CS and 76 had ID. Two cases had attempted vacuum which failed leading to emergency CS.

The age of the patients in both the groups was consistent with the mean age of 25 years. The gestational age varied from $34^{+}$to $43^{+}$weeks. It was found that gestational age as such was not a contributing factor but prematurity or post dated/ post term pregnancy could have added to second stage complications. Both ID (65.8\%) as well as CS $(82.5 \%)$ was found to be maximum in nulliparous patient, which were consistent to studies done by Joseph L Fitzwater et al and Nupur Shah. ${ }^{16,17}$

A larger number of patients who had ID in second stage were those with heart disease. Rheumatic as well as congenital heart diseases contributed to a highest number of patients (18.4\%) who had vacuum delivery to curtail the second stage of labour (prophylactic indication). Consistent to the current study, the study by Hema Priya et al who also stated that about $18 \%$ of the study population had ID to cut short second stage of labour that was further prolonged by addition of epidural anesthesia. ${ }^{18}$

Teenage pregnancy was second risk factor for having a complicated second stage and required ID. This could be attributed to underdeveloped pelvis and poor maternal effort. ${ }^{19}$

Complicated second stage of labour was not seen to be associated with either onset of labour (spontaneous or induced) or PROM, though PROM per se could be a contributing factor for higher rate of $\mathrm{CS}^{20}$

The position of fetal head had an important role to play in the management of second stage of labour. Almost all (100\%) of the fetuses having ID had their head in occipitoanterior position whereas only $49 \%$ had occipitoanterior position during CS. Many studies contribute to the fact that malposition of the fetal head other than occipitoanterior could be one of the leading causes to second stage CS or ID. ${ }^{21,22}$

The maximum indication that required ID during second stage was abnormality in fetal heart rate $(38.1 \%)$ that was followed by meconium stained liquor $(32.8 \%)$. The hypoxic state of second stage, sometimes the abnormal uterine activities or the use of oxytocics bring about hypoxia in the fetus leading to abnormal fetal heart rate and the passage of meconium. Thus active management during second stage with ID can minimize the complications like meconium aspiration syndrome, which probably is universally practiced. ${ }^{23}$
As mentioned earlier prophylactic application of ID in cases with heart diseases to cut short second stage of labour is a norm. About $18.4 \%$ cases had various cardiac lesions and they benefitted from ID. The main aim of applying instrument is the benefit of avoiding hemodynamic fluctuations resulting from pushing during the second stage of labour, which has also been clearly mentioned in study by Pieper PG. ${ }^{24}$

The least numbers of ID was performed for poor maternal effort $(6.5 \%)$ and prolong second stage of labour $(9.2 \%)$. Contradicting the current study, Shrestha BK et al stated that prolonged second stage of labor followed by fetal distress (19.2\%), poor maternal effort $(9.6 \%)$ and to shorten the second stage of labour (8.6\%) respectively, were the commonest indications for application of instruments. ${ }^{25}$

Non descent of head in second stage of labour contributed to the largest number $(77.1 \%)$ undergoing CS, similar to the study done by Babre VM et al where non- descent of the head was the second most common indication leading to CS. Non descent of head in both these studies can be credited to CPD or to DTA. ${ }^{26}$

Very few cases were operated for fetal heart abnormality $(10.5 \%)$, meconium stained liquor $(8.7 \%)$ and only two(3.5\%) had failed vacuum that underwent CS unlike quite a large group that had CS in the same study by Babre VM et al. ${ }^{26}$ This shows that prompt and efficient decision plays a vital role.

The fear of failed ID followed by CS and further more added complications may subject the obstetrician to many litigious medico-legal conditions. Thus this fear usually compels the obstetrician rather to go for emergency CS than to apply ID and fail.

The complications that occurred during ID were $3^{\text {rd }}$ and $4^{\text {th }}$ degree perineal tear. But more so extension of uterine incision was seen in about 29.8\% during second stage CS. This was much more in comparison to the study done by Babre VM et al where the extension reported was just $3.3 \% .{ }^{26}$

Similarly urinary complications like prolonged catheterization $(43.9 \%)$ were more evident in the present study compared to other studies. ${ }^{27}$ Also retention of urine that needed catheterization $(2.6 \%)$ was present in the current study.

Wound infections seen in CS were much more $(14 \%)$ as compared to studies done by Padma Gurung where it was only $(4.8 \%) .{ }^{28}$

Blood loss was more in CS as compared to ID $(p<0.001)$. This could be ascribed to extension of uterine incisions during CS in about a third cases as compared to few cases of $3^{\text {rd }}$ and $4^{\text {th }}$ degree perineal tear during ID contributing to blood loss.

The neonatal weight in both the groups were similar 
with comparable APGAR score in 5 minutes which was more than 7, but the NNU/NICU admission were more in CS than in the ID group. About 50\% needed admission to NNU/NICU in CS group and mostly because of respiratory distress. Even in ID group, respiratory distress contributed to maximum number of admissions. Subgaleal haematoma was seen in a case of attempted vacuum that failed and was delivered by CS. Nevertheless baby recovered well and was discharged. Similar were the finding in the study done by Georgina Davis though the number of cases was comparatively higher. ${ }^{29}$

\section{CONCLUSION}

Despite of few failed attempts at ID, it still proved to be better choice for the management of second stage intervention, as the maternal and neonatal complications were higher following second stage CS. Hence, the correct and timely decision for ID addressing the associated risk factors can lead to favorable outcome.

\section{CONFLICT OF INTEREST}

None declared.

\section{REFERENCES}

1. Bailey P E . The Disappearing Art of Instrumental Delivery: Time to Reverse the Trend. Int J Gynaecol Obstet. 2005 Oct;91(1):89-96.

2. Black C, Kaye JA, Jick H. Cesarean delivery in the United Kingdom:time trends in the general practice research database. Obstet Gynecol. 2005;106:151-5.

3. Eskew PN, Jr, Saywell RM, Jr, Zollinger TW et al. Trends in the frequency of cesarean delivery. A 21-year experience, 1970-1990. J Reprod Med. 1994;39:809-17.

4. Barber EL, Lundsberg $L S$, Belanger $K$ et al. Indications contributing to the increasing cesarean delivery rate. Obstet Gynecol. 2011;118:29-38.

5. Thomas J, Callwood A, Brocklehurst P, Walker J. The National Sentinel Caesarean Section Audit. BJOG. 2000; 107:579-80.

6. WHO Reproductive Health Library. WHO recommendation on definition and duration of the second stage of labour (February 2018). The WHO Reproductive Health Library; Geneva: World Health Organization. Assessed on 3 Sept 2020. http://apps.who.int/ iris/bitstream/10665/250796/8/9789241549912-websupplementeng.pdf?ua=1

7. Lipschuetz M, Cohen SM, Lewkowicz AA, et al.. Prolonged second stage of labor: causes and outcomes. Harefuah. 2018 Nov; 157(11):685-90.[Article in Hebru]

8. Okewole IA, Faiola $S$, Fakounde $A$, et al. The relationship of ethnicity, maternal height and shoe size, and method of delivery. J Obstet Gynaecol. 2011;31:608-11.

9. Benjamin SJ, Daniel AB, Kamath A, Ramkumar V. Anthropometric measurements as predictors of cephalopelvic disproportion:can the diagnostic accuracy be improved? Acta Obstet Gynecol Scand 2012; 91:122-7.

10. Malabarey OT, Balayla J, Abenhaim HA. The effect of pelvic size on cesarean delivery rates:using adolescent maternal age as an unbiased proxy for pelvic size. J Pediatr Adolesc Gynecol. 2012; 25:190-4.

11. Hankins GD, Rowe TF. Operative vaginal delivery--year 2000. Am J Obstet Gynecol. 1996; 175:275-82.

12. Notzon FC, Bergsjø $\mathrm{P}$, Cole $S$ et al. International collaborative effort (ICE) on birth weight, plurality, perinatal, and infant mortality. IV. Differences in obstetrical delivery practice:Norway, Scotland and the United States. Acta Obstet Gynecol Scand. 1991;70:451-60.

13. Zahniser SC, KendrickJS, Franks AL et al. Trends in obstetric operative procedures, 1980 to 1987. Am J Public Health. 1992;82:1340-4.

14. Joseph KS, Young DC, Dodds $L$ et al. Changes in maternal characteristics and obstetric practice and recent increases in primary cesarean delivery. Obstet Gynecol. 2003;102:791-800.

15. Joyce F Sung, Kay I Daniels, Laura Brodzinsky et al. Cesarean Delivery Outcomes After a Prolonged Second Stage of Labor. Am J Obstet Gynecol. 2007 Sep;197(3):306-5.

16. Fitzwate $\mathrm{J} \mathrm{L}$, Owen J, Ankumah $\mathrm{N}$ A et al. Nulliparous Women in the Second Stage of Labor: Changes in Delivery Outcomes Between Two Cohorts From 2000 and 2011. Obstet Gynecol. 2015 Jul; 126(1):81-6.

17. Shah N, Maitra N, Pagi SL. Evaluating role of parity in progress of labour and its outcome using modified WHO partograph. Int J Reprod Contracept Obstet Gynecol. 2016;5(3):860-3

18. Priya HL, Bhandiwad A, Desai N, Kondareddy T. Maternal outcomes of rheumatic heart disease in pregnancy. Int J Reprod Contracept Obstet Gynecol. 2017;6(3):802-6

19. D Rita. Study of feto maternal outcome of Teenage pregnancy at tertiary care hospital. Int J Reprod Contracept Obstet Gynecol. $2017 \mathrm{Jul} ; 6(7): 2841-2845$

20. Xia H, Xilian L, Xiaotian L et al. The clinical management and outcome of term premature rupture of membrane in East China: results from a retrospective multicenter study. Int I Clin Exp Med. 2015; 8(4): 6212-6217.

21. Senécal J, Xiong $X$, Fraser W D . Pushing early or pushing late with epidural study group. Effect of Fetal Position on Second-Stage Duration and Labor Outcome. Obstet Gynecol 2005Apr;105(4):763-72

22. Tan PS, Hwee J K, Loy E. Comparison of Cesarean section and instrumental deliveries at full cervical dilatation: a retrospective review. Singapore Med J. 2019;60(2):75-79

23. Priyadharshini VM, Panicker S. Meconium Stained Liquor and Its Fetal Outcome - Retrospective Study. IOSR-Journal of Dental and Medical Sciences. 2013; 6(2):27-31

24. Piper P.G. The pregnant woman with heart disease: management of pregnancy and delivery. Neth Heart J. 2012 Jan; 20 (1): 33-37.

25. Shrestha BK, Shrestha S, Thapa B. Vacuum Assisted Vaginal Delivery in Singleton Term Pregnancies: Short Term Maternal and Neonatal Outcome in a Tertiary Hospital of Nepal. JLMC; 2016. 4(2): 104-107

26. Babre V M, Bendre K R, Niyogi G. Review of caesarean sections at full dilatation. Int J Reprod Contracept Obstet Gynecol. 2017 ;6(6):2491-2493

27. Moodley J, Devjee J, Khedun SM, Esterhuizen T. Second-stage primary Caesarean deliveries: Are maternal complications increased? SA Fam Pract. 2009;51(4):328-331

28. Gurung P, Malla S, Lama S, Malla A et al. Caesarean Section During Second Stage of Labor in a Tertiary Centre. J Nepal Health Res Counc.2017 May - Aug;15(36): 178-81

29. Davis $G$, Fleming $T$, Ford $K$ et al. Caesarean section at full cervical dilatation. Aust N Z J Obstet Gynaecol. 2015; 55: 565-571. 